\title{
Vaccines versus viruses
}

\section{Influenza virus vaccination for young children living in the US state of New Jersey and who are attending preschool is now compulsory—a mandate that has highlighted a flourishing public mistrust of vaccines.}

T he days are now getting longer in the US, but flu season is not yet over. Fortunately, many people at particular risk from influenza virus infection-young children, the elderly and the immune compromised-can have protected themselves with a flu shot if they or their parents chose to do so. But this year, in New Jersey, children between six months and five years of age are required to be immunized against influenza virus before being allowed to attend day care or preschool. The mandate has angered some parents, particularly those who are persuaded that there is a link between vaccination and autism. They argue that influenza virus vaccination should not be a matter of public health, but rather of personal preference.

The vaccine-autism furor is more than a decade old. In 1998, the British gastroenterologist Andrew Wakefield published a paper in Lancet purporting to show a link between the threein-one shot for measles, mumps and rubella (MMR) and the development of colitis and autism in a small group of previously normal children. The paper triggered a media-fueled panic with far-reaching repercussions: vaccination rates for MMR dropped in the UK, and the incidence of measles infection in children escalated. Around the same time, safety concerns were being voiced in the US about thimerosal - a vaccine preservative that contains a mercury-containing compound.

A link between vaccination and autism has been refuted by the scientific community. Several large epidemiological studies have failed to find a connection between autism and either the MMR vaccine or any vaccine containing thimerosal. The Lancet paper was subsequently deemed to be flawed and its conclusions were retracted by ten of its thirteen authors. Furthermore, thimerosal has been eliminated from most childhood vaccines (apart from some forms of the flu vaccine) since 2001 without a corresponding drop in autism rates - clearly contradictory to the proposed causal effect of the preservative.

And yet some parents of autistic children are convinced that vaccination is responsible for their children's symptoms. One problem is that the etiology of autism is so unclear. It's easy to understand and have sympathy for parents seeking an explanation for their child's autism. But antivaccine groups are vociferous, aggressive and, apparently, also influential, with detrimental effects on public health. If the real triggers for autism were better understood, then parents seeking information on vaccine safety might find the antivaccine lobby less persuasive.

But even if new research breakthroughs provide a clearer understanding of the causes of autism, it's doubtful that vaccines would get better press. Vaccines are like any drug, in that in rare instances they may cause adverse reactions. But, unlike conventional drugs, they are administered to healthy children, and their beneficial effects are invisible to those who receive them. Most parents of young children in the developed world have never seen a child with measles, polio or meningitis - and this is taken for granted. Vaccines have saved countless numbers of lives and prevented the suffering of many more.

To return to influenza, parents weighing the perceived riskto-benefit ratio of immunization may not only be swayed by the antivaccine press; they may also be underestimating the potential dangers of influenza virus infection. These days, it's all too common to complain of symptoms of 'flu' when referring to a few sniffles and a sore throat. The truth is that influenza virus infection can cause serious complications and can even be fatal to certain groups of people_-including young children, whose immune systems are not fully developed. Children attending day care centers and preschools are also effective transmitters of the infection, not only to their playmates but also to their elderly relatives, for whom the consequences of influenza virus infection are often very serious.

Parents in New Jersey still have the choice of whether or not to vaccinate their children against influenza virus-if they are prepared not to send their children to day care or if they have particular religious beliefs that grant them a waiver. The new mandate simply ensures that unvaccinated children will not be putting large numbers of other people at risk. Parents making this choice should do so on the basis of sound information, not suspicion and fear, and they should be fully aware of the risks of the disease the vaccine is intended to prevent. Perhaps it is time for public health officials to start speaking as loudly in support of vaccination as the vaccine detractors speak against it. We should hear more often why childhood immunizations have been one of the greatest medical successes of all time. 